\title{
Editorial
}

\section{COOPERATION IN SCIENCE}

\author{
Marian Adamkov \\ Department of Histology and Embryology, Jessenius Faculty of Medicine, Comenius \\ University in Bratislava, Martin, Slovakia
}

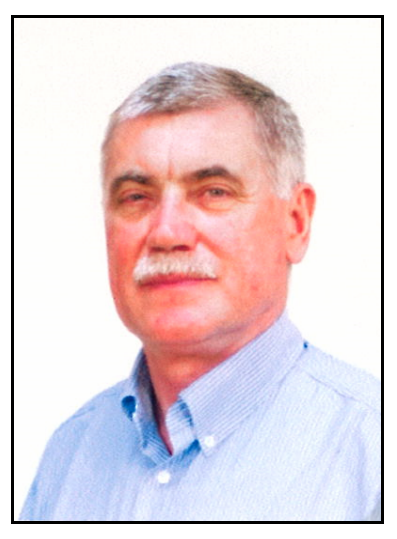

Over the past decades there is an evident trend of integration of medical research. The known borders between individual subject fields gradually disappear. The aim is to achieve better understanding of the biocomplexity of healthy and pathologically changed cells, tissues and organs. The human body is a complex organism, in the making of which there is a close cooperation of its individual parts at various levels of organization. Cells combine to form tissues, tissues to form organs, organs to form systems and the systems ultimately enter into the anatomical arrangements of the human body. Biological systems carry out specific functions. Nowadays scientists in medicine are confronted with enormous accumulation of knowledge and they are not able to follow all of the new information. Therefore, there is a tendency to overspecialize or subspecialize in every single medical field. Logically, growing activities in cooperation between specialists from different branches of interest are noticed.

Every field of study has also its natural limitations and needs complementation from other scientific approaches. Moreover, we are all in one way or the other victims of the trend of narrow specialization. We often assess biological subjects with our subjective (e.g. histological or anatomical) eye overlooking possibilities and potentials of contributions of other scientific disciplines. However, new pieces of knowledge from other disciplines may help significantly to resolve our own scientific problems. In addition, it can also make our research more effective.

Many scientists are still entrapped in their own medical subject as a result of disintegration due to narrow specialization and are often not completely aware of the real biocomplexity of cells, tissues and organs as well as organisms. As an opposite example, we can take the morphological subject Histology. Histology stands at the crossroad between gross anatomy and physiology. This subject acts as an integrative element between them. While teaching is mainly limited to functional histology, research activities focus on micromorphology of life substances in general. Histology, in close cooperation with physiology, biochemistry and molecular biology, provides new important data concerning the subcellular or intercellular localization of single molecules, as well as cells and united cell structures in human body. For example, the study of apoptosis was first based on cell morphology only using light and electron microscopy. Recently, marked progress in biochemistry, immunology, molecular biology and genetics provided the researchers of apoptosis with various tools for precise detection of apoptotic cells, such as immunohistochemical methods, agarose gel electrophoresis, flow cytometry, etc. Needless to say, that methodological background serves as a common denominator in scientific cooperation.

Without mutual cooperation, today's modem science would be unthinkable. 